
ГИПОПАРАТИРЕОЗЕ: СЕРИЯ КЛИНИЧЕСКИХ СЛУЧАЕВ

\author{
() Т.М. Афонасьева, Е.В. Ковалева*, А.К. Еремкина, Н.Г. Мокрышева
}

Национальный медицинский исследовательский центр эндокринологии, Москва, Россия

Идиопатический гипопаратиреоз - редкое эндокринное заболевание, устанавливаемое при исключении всех возможных причин развития гипопаратиреоза.

На сегодняшний день данные о распространенности идиопатического гипопаратиреоза ограничиваются рядом локальных исследований среди населения отдельных стран. Разнообразие клинических проявлений гипопаратиреоза, схожесть с проявлениями ряда других патологий, в частности, эпилепсии, значимо удлиняют путь пациента до постановки правильного диагноза и назначения патогенетической терапии.

Учитывая осведомленность клиницистов различного профиля о хирургическом гипопаратиреозе и редкость идиопатической формы в клинической практике, становится понятным, что отсутствие хирургического вмешательства на органах шеи в анамнезе практически исключает гипопаратиреоз из дифференциального поиска.

При подтверждении гипопаратиреоза нехирургической этиологии необходимо применение всех известных на сегодняшний день диагностических ресурсов для уточнения этиологии заболевания.

В статье представлена серия клинических случаев, отражающих сложность диагностики и особенности течения идиопатического гипопаратиреоза. Рассмотрены имеющиеся в настоящее время методы диагностики, в частности, определение уровня интерферона-омега при подозрении на аутоиммунный полигландулярный синдром 1 типа как наиболее распространенную причину нехирургического гипопаратиреоза.

КЛЮЧЕВЫЕ СЛОВА: идиопатический гипопаратиреоз; аутоиммунный полигландулярный синдром 1 типа; послеоперационный гипопаратиреоз; диффреренциальная диагностика.

\title{
DIFFERENTIAL DIAGNOSIS OF IDIOPATHIC HYPOPARATHYROIDISM: A CASE SERIES
}

(c) Tatiana M. Afonasyeva, Elena V. Kovaleva*, Anna K. Eremkina, Natalia G. Mokrysheva

Endocrinology Research Centre, Moscow, Russia

Idiopathic hypoparathyroidism is a rare endocrine disease diagnosed when all possible causes of hypoparathyroidism are excluded.

Data on the prevalence of idiopathic hypoparathyroidism is limited, there are several local studies in some countries. The variety and non-specificity of the hypoparathyroidism symptoms, similarity to other diseases (eg. epilepsy), causes the misdiagnosis and delay in pathogenetic therapy.

Idiopathic hypoparathyroidism is rare. Therefore, the absence of neck surgery in the anamnesis practically excludes hypoparathyroidism from the diagnostic search. Moreover, it is necessary to rule out all known causes of decreased PTH hypoparathyroidism to determine the form of non-surgical hypoparathyroidism.

We present a case series of idiopathic hypoparathyroidism, which demonstrates the difficulty in diagnosis, and the clinical features of the disease. The article covers also the problem of modern diagnostic approaches including the determination of the interferon-omega for patients with suspected autoimmune polyglandular syndrome type 1 as the most common cause of nonsurgical hypoparathyroidism.

KEYWORDS: idiopathic hypoparathyroidism; autoimmune polyglandular syndrome type l; postsurgical hypoparathyroidism; differential diagnostics.

\section{АКТУАЛЬНОСТЬ}

Гипопаратиреоз представляет собой редкое эндокринное заболевание, характеризующееся недостаточностью паратиреоидного гормона (ПТГ) и нарушением фосфорно-кальциевого обмена с развитием гипокальциемии и гиперфосфатемии. Распространенность в общей популяции составляет порядка 0,2-0,3\% [1]. Среди основных форм заболевания первое место (около 75\% случаев) занимает послеоперационный гипопаратиреоз, возника- ющий после хирургических вмешательств на органах шеи. Оставшиеся 25\% приходятся на иные формы [2], при этом истинная распространенность нехирургического гипопаратиреоза остается неизвестной. Информация ограничивается результатами немногочисленных исследований, проведенных в ряде стран с включением различных этнических групп. Так, распространенность нехирургического гипопаратиреоза в Японии составляет 0,7 на 100000 человек, в Корее - 1,1 на 100 000, в Дании - 2,3 на 100 000, в США - 4,8 на 100000 [3]. 


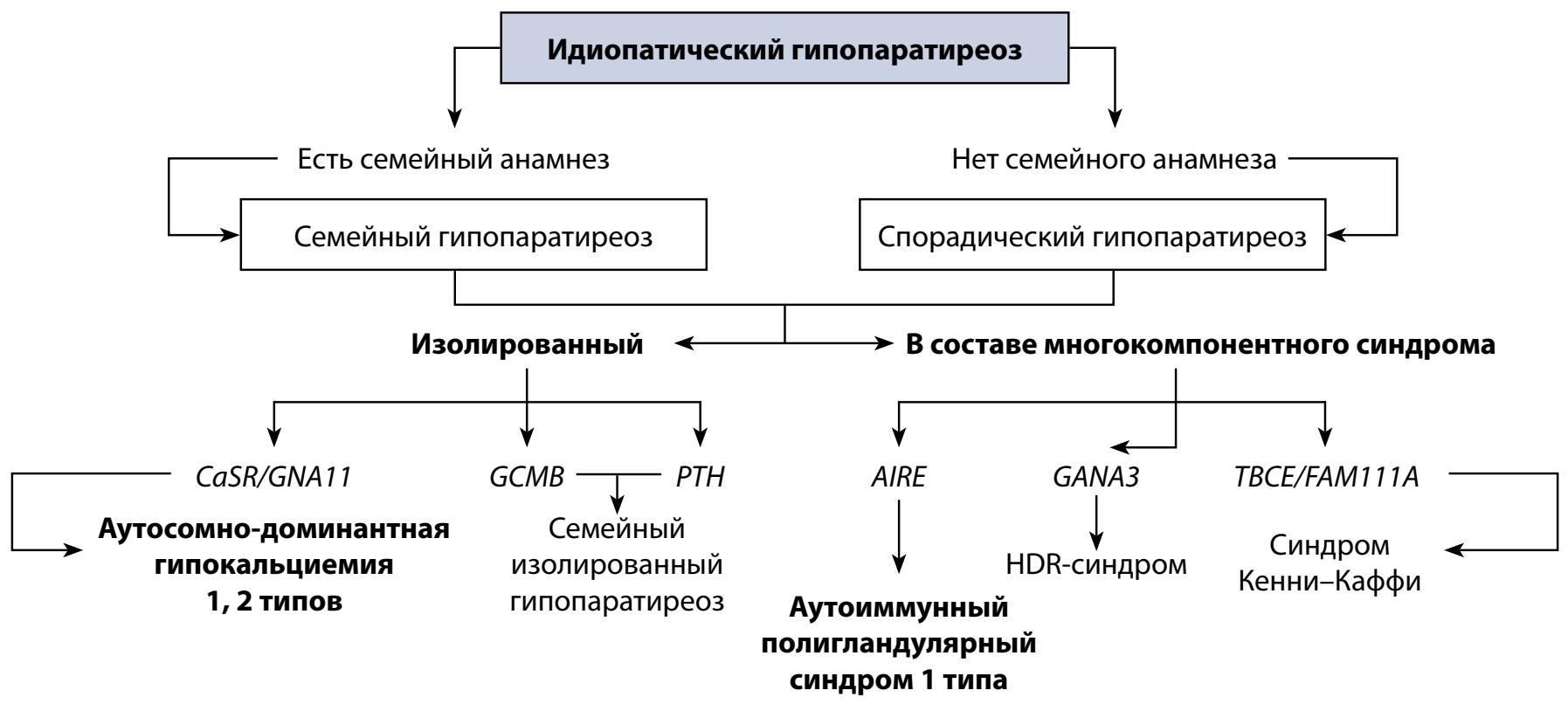

Рисунок 1. Диагностический поиск при идиопатическом гипопаратиреозе [4].

Нехирургический гипопаратиреоз, как правило, ассоциирован с аутоиммунными или генетическими причинами (рис. 1) [4]. Аутоиммунный гипопаратиреоз в основном развивается в составе аутоиммунного полигландулярного синдрома 1 типа (АПС 1 типа) [5, 6]. Среди наиболее частых генетических причин заболевания также можно выделить аутосомно-доминантную гипокальциемию, вызванную активирующей мутацией в гене кальций-чувствительного рецептора (CASR) [7].

Диагноз идиопатического гипопаратиреоза устанавливается после исключения основных причин развития заболевания, в том числе с помощью медико-генетического консультирования. По данным ряда авторов, распространенность идиопатического гипопаратиреоза составляет от 5,5-9 случаев на 1 млн человек [8-12]. По результатам эпидемиологического исследования норвежской популяции, около трети случаев нехирургического гипопаратиреоза остаются идиопатическими [3], а в Дании и Корее их частота может достигать 47 и 95,9\% соответственно [2,9].

В статье представлен ряд клинических случаев, отражающих сложность диагностики и особенности клинической картины идиопатического гипопаратиреоза.

\section{ОПИСАНИЕ КЛИНИЧЕСКИХ СЛУЧАЕВ}

\section{Клинический случай 1.}

Пациент М., 37 лет. Из анамнеза известно, что в 21 год впервые отметил появление парестезий и болей в нижних конечностях при длительном вынужденном положении (рис. 2). В течение последующих нескольких лет дважды (в возрасте 23 и 26 лет) возникали генерализованные тонико-клонические судороги. После повторного судорожного эпизода пациент был госпитализирован в терапевтическое отделение по месту жительства. При обследовании впервые выявлена гипокальциемия (кальций общий 1,66-1,9 ммоль/л, референсный интервал (РИ) 2,15-2,55), которая была купирована парентеральным введением препаратов кальция. Дальнейшего диагностического поиска не проводилось. Уровень ПТГ был определен только при повторной госпитализации с симптомами острой гипокальциемии и составил 0,4 пмоль/л (РИ 1,6-6,9). В биохимическом анализе крови сохранялось значимое снижение уровня общего кальция (1,88 ммоль/л), зафиксированы гиперфосфатемия $(1,98$ ммоль/л, РИ 0,74-1,52) и гипокальциурия $(0,4$ ммоль/сут, РИ 2,5-6,2). Был диагностирован гипопаратиреоз, назначена медикаментозная терапия препаратами кальция (кальция карбонатом 1000 мг/сут) и активным метаболитом витамина D (альфакальцидолом 2 мкг/сут). B последующем подобранное лечение пациент принимал эпизодически, приступов тонико-клонических судорог не повторялось.

В феврале 2020 г. поступил в отделение патологии околощитовидных желез (ОПОЩЖ) ФГБУ «НМИЦ эндокринологии» Минздрава России с жалобами на «мурашки», парестезии, периодически возникающие судороги в руках и ногах, общий спазм мышц, повышенную утомляемость и снижение работоспособности. При физикальном осмотре кожные покровы и видимые слизистые оболочки бледно-розовые, проявлений кандидоза не обнаружено. Гемодинамические показатели стабильны (артериальное давление 125/70 мм рт. ст.; пульс 68 уд/ мин). Симптомы Хвостека и Труссо слабоположительные. На фоне приема постоянной терапии (альфакальцидол 3 мкг/сут, карбонат кальция 1500 мг/сут, колекальциферол 600 ME/сут) была выявлена гипокальциемия (альбумин-скорректированный кальций 1,78 ммоль/л) в сочетании с гиперфосфатемией (1,55 ммоль/л) и нормокальциурией (3,86 ммоль/сут), снижением уровня ПтГ до 3,2 пг/мл (РИ 15-65), что подтверждало диагноз гипопаратиреоза. Кальций-креатининовый индекс (calcium:creatinine ratio, CCR) - 0,03 (нормальный РИ $<0,14$; гиперкальциурия при CCR >0,20) [13]. Гипомагниемия исключалась (магний 0,7 ммоль/л, РИ 0,7-1,05).

Учитывая отсутствие хирургического вмешательства на органах шеи в анамнезе, волнообразное течение заболевания с чередованием периодов декомпенсации и относительного благополучия, а также относительно молодой возраст манифестации гипопаратиреоза (в 21 год), 

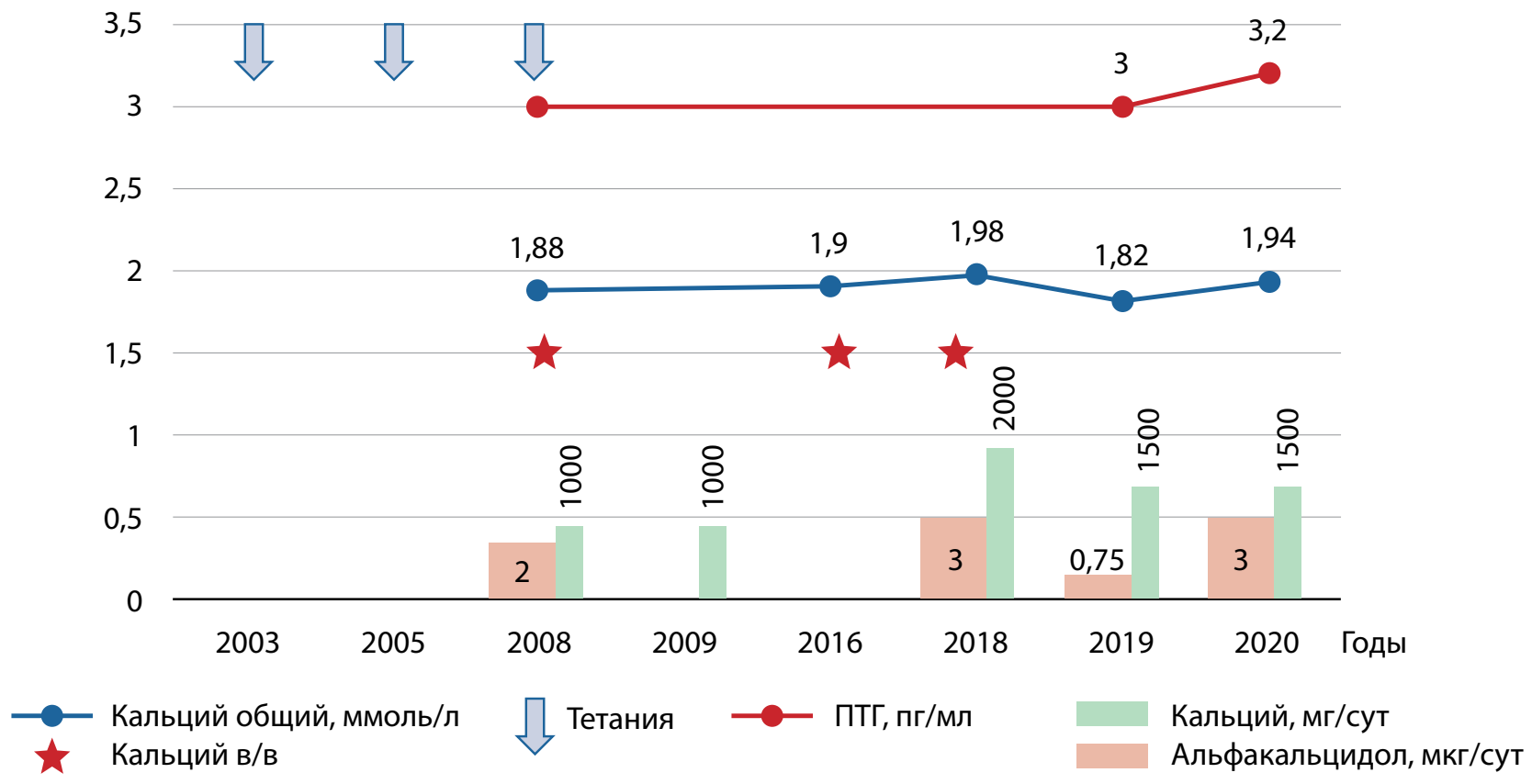

Рисунок 2. Динамика изменений клинической и лабораторной картины.

Референсный интервал для паратиреоидного гормона (ПТГ) 15-65 пг/мл, для кальция общего - 2,15-2,55 ммоль/л.

проведено обследование на наличие АПС 1 типа. Исключена первичная надпочечниковая недостаточность (кортизол крови утром 564 нмоль/л, РИ 171-536), антитела к интерферону- $\omega$ (IFN- $\omega$ ) отрицательные, при полном секвенировании гена AIRE мутаций не обнаружено. Принимая во внимание отсутствие отягощенного семейного анамнеза и гиперкальциурии, аутосомно-доминантная гипокальциемия была исключена из дифференциально-диагностического поиска. Таким образом, выявленные изменения были классифицированы как идиопатический гипопаратиреоз с рекомендацией дальнейшего динамического наблюдения и обследования. В рамках госпитализации проведена коррекция терапии: увеличена доза альфакальцидола до 3,5 мкг/сут, карбоната кальция до 2000 мг/сут, с положительной динамикой лабораторных показателей.

\section{Клинический случай 2.}

Пациентка Б., 21 год. Из анамнеза известно, что в младенчестве отмечались судороги, расцениваемые как фебрильные, которые в дальнейшем, однако, возникали и на фоне нормальной температуры тела с частотой 2-3 раза в год и носили тонико-клонический характер. По данным электроэнцефалографии (ЭЭГ) неоднократно выявлялась эпилептиформная активность. Получала противосудорожную терапию препаратами вальпроевой кислоты и топирамата.

В возрасте 15 лет были впервые оценены показатели фосфорно-кальциевого обмена: выявлены значимая гипокальциемия (кальций общий 1,79 ммоль/л), гиперфосфатемия (2,32 ммоль/л), низкий уровень ПТГ (9 пг/мл), что позволило установить диагноз гипопаратиреоза. Подобрана терапия кальцитриолом 1,75 мкг/сут. Проведена мультиспиральная компьютерная томография головного мозга: диагностирован синдром Фара.

С 16 лет пациентка наблюдается в ФГБУ «НМИЦ эндокринологии» Минздрава России. Для исключения АПС
1 типа проведено полное секвенирование гена AIRE мутаций не выявлено, IFN- $\omega$ отрицательный. Пороков развития не отмечено, так же как и других возможных сопутствующих патологий в рамках синдромальных форм гипопаратиреоза. У кровных родственников нарушений функции ОЩЖ не обнаружено.

Последняя госпитализация в ОПОЩЖ в октябре 2019 г. Поступила с жалобами на судороги в икроножных мышцах, возникающие при резких движениях, воздействии холода и в ночное время суток с периодичностью до 4 раз в неделю; на дискомфорт в области сердца, снижение памяти, головные боли, нарушение менструальной функции, задержку стула до 3-4 дней. При физикальном осмотре: кожные покровы и видимые слизистые оболочки бледно-розовые, проявлений кандидоза не обнаружено. Гемодинамические показатели стабильны (артериальное давление 110/70 мм рт. ст.; пульс 72 уд/мин). Симптомы Хвостека и Труссо отрицательные. При обследовании выявлена легкая гипокальциемия (2,03 ммоль/л) в сочетании с выраженной гиперфосфатемией (2,09 ммоль/л), нормокальциурия (5,78 ммоль/сут). CCR 0,0275. При проведении дополнительного обследования исключены сочетанные эндокринная и соматическая патологии. В исходе госпитализации достигнута клиническая и лабораторная компенсация заболевания на фоне лечения: кальция карбонат 1500 мг/сут, кальцитриол 1,75 мкг/сут.

Клинический случай 3.

Пациент В., 51 год. Из анамнеза известно, что в 9-летнем возрасте проведено удаление кисты в области шеи (медицинской документации не предоставлено). По выпискам из амбулаторной карты от 2012 г. (возраст 43 года) уровень кальция был на нижней границе нормы.

С конца января 2020 г. родственники отметили изменения поведения по типу агрессии, дезориентации в пространстве. Неделей раньше наблюдалась гипертермия до $39^{\circ} \mathrm{C}$, сопровождавшаяся появлением высыпаний 
на руках и внутренней поверхности бедер. 01.02.2020 г. бригадой СМП пациент был госпитализирован с впервые возникшим судорожным приступом. При обследовании в инфекционном стационаре диагностирована тяжелая форма генерализованной бактериальной инфекции, неуточненной этиологии; токсико-дисциркуляторная энцефалопатия; моторная эфферентная афазия; тетания. Исследование уровня кальция крови не проводилось. По результатам МРТ головного мозга от 09.02.2020 г. визуализированы мелкие единичные очаги в полушариях большого мозга, вероятно, сосудистого генеза. Проводилась массивная антибактериальная терапия. После выписки из стационара судороги возобновились.

29.02.2020 г. - эпизод потери сознания, в связи с чем повторно был госпитализирован уже в неврологическое отделение. Проводилось расширенное инструментальное обследование. Острая патология исключена по данным КТ головного мозга. При ЭЭГ патологической биоэлектрической активности не фиксировалось. В связи с впервые выявленными гипокальциемией (кальций общий 1,03 ммоль/л) и снижением уровня ПТГ (менее 3 пг/мл) пациент был переведен в эндокринологическое отделение. Для купирования острой гипокальциемии проводилась инфузионная терапия глюконатом кальция, с последующим назначением альфакальцидола 1 мкг/сут, карбоната кальция 1000 мг/сут, однако несмотря на отсутствие достижения целевых значений кальциемии дозы пероральных препаратов в стационаре не менялись. Несмотря на это, у пациента был отмечен регресс неврологической симптоматики в виде восстановления когнитивных функций, эмоционального статуса, тем не менее судороги и парестезии сохранялись. Терапия гипопаратиреоза в дальнейшем корректировалась на амбулаторном этапе.

Впервые поступил в ОПОЩЖ в мае 2020 г. с жалобами на легкие парестезии в области лица, периодические головные боли, общую слабость. Симптомы Хвостека и Труссо отрицательные. Терапия при поступлении по схеме: альфакальцидол 3 мкг/сут, карбонат кальция 2000 мг/сут, колекальциферол 1500 МЕ/сут. По результатам лабораторных анализов подтверждена компенсация заболевания с достижением целевых значений: альбумин-скорректированный кальций 2,17 ммоль/л, фосфор 1,52 ммоль/л, кальций в суточной моче 5,86 ммоль/сут (РИ 2,5-7,5). CCR 0,0423. Учитывая поздний возраст манифестации и отсутствие других клинических симптомов, ассоциированных с наследственными формами гипопаратиреоза, генетическое тестирование не проводилось.

\section{ОБСУЖДЕНИЕ}

Представленные клинические случаи демонстрируют сложности в своевременной постановке диагноза нехирургического гипопаратиреоза. Ввиду большого количества неспецифических симптомов диагностика данного заболевания напрямую зависит от первоначального определения уровня кальция сыворотки. К сожалению, данный электролит не входит в стандартный биохимический анализ крови, что значимо удлиняет путь пациента до правильного диагноза и лечения. Это, в свою очередь, существенно ухудшает прогноз и качество жизни больных.
Принимая во внимание тот факт, что послеоперационный гипопаратиреоз является наиболее распространенной формой заболевания, становится понятным, почему отсутствие хирургического вмешательства на органах шеи в анамнезе зачастую исключает гипопаратиреоз из дифференциально-диагностического поиска и «направляет» клиницистов в сторону других патологий со сходной клинической картиной. В Китае частота ошибочно выставленных диагнозов, сохранявшихся за пациентами с идиопатическим гипопаратиреозом в течение нескольких лет, составила 29,5\% (период наблюдения с 1994 по 2013 гг.). Наиболее часто устанавливались эпилепсия (60\%), далее следовали неврозы и психические расстройства (6,64\%). Реже дифференциальная диагностика проводилась с такими заболеваниями, как полимиозит, туберкулезный менингит, фебрильные судороги, паразитозы, дефицит витамина К и другие [14].

В первых двух клинических случаях в виду относительно молодого возраста манифестации заболевания проводилось исключение аутоиммунного гипопаратиреоза в рамках АПС 1 типа (вторая по частоте причина гипопаратиреоза). Несмотря на то, что проявление «классических» компонентов АПС 1 типа чаще наблюдается в течение первых 10 лет жизни, в литературе встречается описание и более поздней манифестации синдрома [15]. Помимо традиционного секвенирования гена AIRE, для установления диагноза АПС 1 типа в России и мире используется определение антител к IFN- $\omega$ [16-18]. В представленных клинических случаях применялись оба метода диагностики, при этом АПС 1 типа не был подтвержден.

Во 2 клиническом случае симптомы гипопаратиреоза маскировались проявлениями эпилепсии, что не позволяло вовремя диагностировать гипокальциемию и начать патогенетическое лечение. В данном случае обращает на себя внимание отсутствие настороженности специалистов в поиске редких эндокринных заболеваний.

В 3 клиническом случае гипокальциемические судороги изначально были идентифицированы как фебрильные на фоне тяжелого течения генерализованной бактериальной инфекции. Случившаяся в дальнейшем потеря сознания, с учетом возраста пациента, расценена как острая неврологическая патология. Лишь при исключении последней, пациент был направлен в эндокринологический стационар, где был установлен диагноз и начата адекватная терапия. Важно, что на фоне достижения околоцелевых значений кальциемии неврологическая симптоматика полностью регрессировала. Яркая клиническая картина на фоне инфекционного процесса, отсутствие убедительных анамнестических данных (операция в области шеи в 9-летнем возрасте), заставили клиницистов исключить, в первую очередь, общеизвестные жизнеугрожающие состояния со сходной клинической картиной, не принимая во внимание гипопаратиреоз.

Гипокальциемия является широко распространенным явлением (до 88\%) у тяжелых больных из отделений интенсивной терапии, в том числе по причине инфекционных заболеваний. Тем не менее, механизмы ее развития до конца не изучены, основные гипотезы включают в себя дефицит 1,25-дигидроксивитамина D3, повышенный уровень кальцитонина при синдроме системной 
воспалительной реакции или избыточной секреции провоспалительных цитокинов (в том числе интерлейкины 1 и 6 типов, фактор некроза опухоли альфа). Тем не менее, гипокальциемия в таких случаях обратима и связана с купированием основного заболевания [19]. В литературе нет прямых доказательств повреждения ОЩЖ на фоне сепсиса. Более того, в пилотном исследовании Carlstedt et al. было показано, что среди пациентов из блока интенсивной терапии (в том числе госпитализированных по поводу сепсиса) чаще наблюдалась гипокальциемия и повышенный уровень ПТГ, последнее ассоциировано с плохим неблагоприятным исходом [20].

В литературе, описаны случаи отсроченного развития послеоперационного гипопаратиреоза спустя 3,5 [21], 10,15 [22], 19 [23] лет и 33 года [24]. Максимальный описанный период отсроченного развития послеоперационного гипопаратиреоза в исходе хирургического вмешательства на органах шеи составил 36 лет [25]. Данный феномен объясняется возрастным нарушением кровоснабжения оставшейся ткани ОЩЖ, что приводит к декомпенсации фосфорно-кальциевого обмена и манифестации заболевания. Учитывая отсутствие доказательных данных об ассоциации септического воспаления с повреждением ОЩЖ и как следствие развитием гипопаратиреоза, отсроченный послеоперационный гипопаратиреоз представляется наиболее вероятной причиной развития заболевания в нашем наблюдении, при этом манифестация произошла через 42 года после операции.

\section{ЗАКЛЮЧЕНИЕ}

Несмотря на накопленный опыт по ведению пациентов с гипопаратиреозом и имеющиеся диагностические ресурсы для его выявления, большой процент установленных случаев заболевания остаются идиопатическими.
Множественные клинические проявления гипопаратиреоза зачастую скрывают заболевание под масками других патологий, что удлиняет время постановки окончательного диагноза и отдаляет назначение патогенетической терапии.

При подтверждении гипопаратиреоза нехирургической этиологии необходимо применение всех известных на сегодняшний день диагностических инструментов для уточнения этиологии заболевания.

\section{ДОПОЛНИТЕЛЬНАЯ ИНФОРМАЦИЯ}

Источники финансирования. Данное исследование выполнено в рамках государственного задания «Всероссийский реестр пациентов с хроническим гипопаратиреозом как основа оптимизации и внедрения персонализированного подхода для улучшения качества оказания медицинской помощи населению Российской Федерации», регистрационный номер НИОКТРАААА-А20-120011790168-2.

Конфликт интересов. Авторы декларируют отсутствие явных и потенциальных конфликтов интересов, связанных с содержанием настоящей статьи.

Участие авторов. Афонасьева Т.М. - получение, анализ данных и интерпретация результатов, написание статьи; Ковалева Е.В. - получение, анализ данных и интерпретация результатов, внесение в рукопись существенной правки с целью повышения научной ценности статьи; Еремкина А.К. - получение, анализ данных и интерпретация результатов, внесение в рукопись существенной правки с целью повышения научной ценности статьи; Мокрышева Н.Г. - существенный вклад в концепцию и дизайн исследования, внесение в рукопись существенной правки с целью повышения научной ценности статьи. Все авторы одобрили финальную версию статьи перед публикацией, выразили согласие нести ответственность за все аспекты работы, подразумевающую надлежащее изучение и решение вопросов, связанных с точностью или добросовестностью любой части работы.

Согласие пациента. Пациенты добровольно подписали информированное согласие на публикацию персональной медицинской информации в обезличенной форме.

\section{СПИСОК ЛИТЕРАТУРЫ | REFERENCES}

1. Умярова Д.Ш., Гребенникова Т.А., Тарбаева Н.В., Белая Ж.Е. Лечение тяжелого идиопатического гипопаратиреоза на примере клинического случая // Ocmeonoроз и остеопатии. - 2018. T. 21. — №2. - C. 36-40. [Umiarova DS, Grebennikova TA, Tarbaeva NV, Belaya ZE. Treatment of severe idiopathic hypoparathyroidism: a case report. Osteoporosis and bone diseases. 2018;21 (2):36-40. (In Russ.)]. doi: https://doi.org/10.14341/osteo9878

2. Kim SH, Rhee Y, Kim YM, et al. Prevalence and complications of nonsurgical hypoparathyroidism in Korea: A nationwide cohort study. PLoS One. 2020;15(5):e0232842. doi: https://doi.org/10.1371/journal.pone.0232842

3. Astor MC, Lovas K, Debowska A, et al. Epidemiology and Health-Related Quality of Life in Hypoparathyroidism in Norway. J Clin Endocrinol Metab. 2016;101(8):3045-3053. doi: https://doi.org/10.1210/jc.2016-1477

4. Cianferotti L, Brandi ML. Chapter 50

The Molecular Genetics of Hypoparathyroidism. Ed. by Bilezikian J.P. San Diego: Academic Press; 2015. P. 719-736. doi: https://doi.org/10.1016/B978-0-12-397166-1.00050-3

5. Orlova EM, Bukina AM, Kuznetsova ES, et al. Autoimmune polyglandular syndrome type 1 in Russian patients: clinical variants and autoimmune regulator mutations. Horm Res Paediatr. 2010;73(6):449-457. doi: https://doi.org/10.1159/000313585

6. Wolff AS, Erichsen MM, Meager A, et al. Autoimmune polyendocrine syndrome type 1 in Norway: phenotypic variation, autoantibodies, and novel mutations in the autoimmune regulator gene. J Clin Endocrinol Metab. 2007;92(2):595-603. doi: https://doi.org/10.1210/jc.2006-1873
7. Raue F, Pichl J, Dörr HG, et al. Activating mutations in the calcium-sensing receptor: genetic and clinical spectrum in 25 patients with autosomal dominant hypocalcaemia a German survey. Clin Endocrinol (Oxf). 2011;75(6):760-765. doi: https://doi.org/10.1111/j.1365-2265.2011.04142.x

8. Nakamura Y, Matsumoto T, Tamakoshi A, et al. Prevalence of idiopathic hypoparathyroidism and pseudohypoparathyroidism in Japan. J Epidemiol. 2000;10(1):29-33. doi: https://doi.org/10.2188/jea.10.29

9. Underbjerg L, Sikjaer T, Mosekilde L, et al. The Epidemiology of Nonsurgical Hypoparathyroidism in Denmark: A Nationwide Case Finding Study. J Bone Miner Res. 2015;30(9):1738-1744. doi: https://doi.org/10.1002/jbmr.2501

10. Clarke BL, Brown EM, Collins MT, et al. Epidemiology and diagnosis of hypoparathyroidism. J Clin Endocrinol Metab. 2016;101(6):2284-2299. doi: https://doi.org/10.1210/jc.2015-3908

11. Powers J, Joy K, Ruscio A, et al. Prevalence and incidence of hypoparathyroidism in the United States using a large claims database. J Bone Miner Res. 2013;28(12):2570-2576. doi: https://doi.org/10.1002/jbmr.2004

12. Mannstadt M, Bilezikian JP, Thakker RV, et al. Hypoparathyroidism. Nat Rev Dis Primers. 2017;3:17055. doi: https://doi.org/10.1038/nrdp.2017.55

13. Foley KF, Boccuzzi L. Urine Calcium: Laboratory Measurement and Clinical Utility. Lab Med. 2010;41(11):683-686. doi: https://doi.org/10.1309/LM9SO94ZNBHEDNTM 
14. Li L L Yang H, Li J, et al. Misdiagnosis of idiopathic hypoparathyroidism: A case report and literature review. Medicine (Baltimore). 2018;97(9):e9884. doi: https://doi.org/10.1097/MD.0000000000009884

15. Трошина В.В., Гребенникова Т.А., Белая Ж.Е. Клинический случай поздней манифестации первичной надпочечниковой недостаточности в рамках аутоиммунного полигландулярного синдрома 1-го типа // Альманах клинической медицины. 2019. — T. 47. — №2. - C.175-179. [Troshina W, Grebennikova TA, Belaya ZhE. A case of late manifestation of primary adrenal insufficiency in the autoimmune polyglandular syndrome type 1. Almanac of Clinical Medicine. 2019;47(2):175-179. (In Russ.)]. doi: https://doi.org/10.18786/2072-0505-2019-47-017

16. Meloni A, Furcas M, Cetani F, et al. Autoantibodies against type I interferons as an additional diagnostic criterion for autoimmune polyendocrine syndrome type I. J Clin Endocrinol Metab. 2008;93(11):4389-4397. doi: https://doi.org/10.1210/jc.2008-0935

17. Oftedal BE, Wolff AS, Bratland E, et al. Radioimmunoassay for autoantibodies against interferon omega; its use in the diagnosis of autoimmune polyendocrine syndrome type I. Clin Immunol. 2008;129(1):163-169. doi: https://doi.org/10.1016/j.clim.2008.07.002

18. Созаева Л.С. Новые иммунологические методы диагностики аутоиммунного полиэндокринного синдрома 1-го типа // Проблемы Эндокринологии. — 2015. - Т.61. — №3 C.43-46. [Sozaeva LS. The new immunological methods for diagnostics of type 1 autoimmune polyendocrine syndrome.
Problems of Endocrinology. 2015;61(3):43-46. (In Russ.)]. doi: https://doi.org/10.14341/probl201561343-46

19. Zivin JR, Gooley T, Zager RA, Ryan MJ. Hypocalcemia: a pervasive metabolic abnormality in the critically ill. Am J Kidney Dis. 2001;37(4):689-698. doi: https://doi.org/10.1016/s0272-6386(01)80116-5

20. Carlstedt F, Lind L, Rastad J, et al. Parathyroid hormone and ionized calcium levels are related to the severity of illness and survival in critically ill patients. Eur J Clin Invest. 1998;28(11):898-903. doi: https://doi.org/10.1046/j.1365-2362.1998.00391.x

21. Simões CA, Costa MK, Comerlato LB, et al. A Case of "Late" Postsurgical Hypoparathyroidism. Case Rep Endocrinol. 2017;2017:1-2. doi: https://doi.org/10.1155/2017/3962951

22. Kamath SD, Rao BS. Delayed Post-Surgical Hypoparathyroidism: The Forgotten Chameleon! J Clin Diagn Res. 2017;11(2):OD07-OD09. doi: https://doi.org/10.7860/JCDR/2017/23609.9260

23. Cox RE. Hypoparathyroidism: an unusual cause of seizures. Ann Emerg Med. 1983;12(5):314-315. doi: https://doi.org/10.1016/s0196-0644(83)80517-4

24. Blanchard BM. Focal Hypocalcemic Seizures 33 Years After Thyroidectomy. Archives of Internal Medicine. 1962;110(3):382-385. doi: https://doi.org/10.1001/archinte.1962.03620210106018

25. Bellamy RJ. Kendall-Taylor P. Unrecognized hypocalcaemia diagnosed 36 years after thyroidectomy. Journal of the Royal Society of Medicine. 1995;88(12):690-691. doi: https://doi.org/10.1155/2020/1735351

\section{ИНФОРМАЦИЯ ОБ АВТОРАХ [AUTHORS INFO]:}

*Ковалева Елена Владимировна [Elena V. Kovaleva, MD]; адрес: Россия, 117036, Москва, ул. Дм. Ульянова, д. 11 [address: 11 Dm. Ulyanova street, 117036 Moscow, Russia]; ORCID: https://orcid.org/0000-0002-9258-2591; eLibrary SPIN: 7387-6791; e-mail: elen.v.kovaleva@gmail.com

Афонасьева Татьяна Михайловна, ординатор [Tatiana M. Afonasyeva, resident]; ORCID: https://orcid.org/0000-0003-0212-8158; eLibrary SPIN: 3928-7138; e-mail: tmafonya@mail.ru

Еремкина Анна Константиновна, К.м.н. [Anna K. Eremkina, MD, PhD]; ORCID: https://orcid.org/0000-0001-6667-062X; Researcher ID: R-8848-2019; Scopus author ID: 57197775339; eLibrary SPIN: 884a8-2660; e-mail: a.lipatenkova@gmail.com Мокрышева Наталья Георгиевна, д.м.н., професcop [Natalia G. Mokrysheva, MD, PhD, Professor]; ORCID: https://orcid.org/0000-0002-9717-9742; eLibrary SPIN: 5624-3875; e-mail: mokrisheva.natalia@endocrincentr.ru

*Автор, ответственный за переписку / Corresponding author.

\section{ЦИТИРОВАТЬ:}

Афонасьева Т.М., Ковалева Е.В., Еремкина А.К., Мокрышева Н.Г. Этапы дифференциальной диагностики при идиопатическом гипопаратиреозе: серия клинических случаев // Ожирение и метаболизм. - 2021. — Т. 18. — №3. - C. 276-281. doi: https://doi.org/10.14341/omet12726

\section{TO CITE THIS ARTICLE:}

Afonasyeva TM, Kovaleva EV, Eremkina AK, Mokrysheva NG. Differential diagnosis of idiopathic hypoparathyroidism: a case series. Obesity and metabolism. 2021;18(3):276-281. doi: https://doi.org/10.14341/omet12726 